Cahiers
de a Recherche
Fourles Droits
Fondamentaux

Cahiers de la recherche sur les droits fondamentaux

$15 \mid 2017$

Le corps humain saisi par le droit : entre liberté et propriété

\title{
Le corps des personnes détenues : de l'objet de punition au respect de la personne
}

The Body of a Prisoner: A Transition from an Object of Punishment to Respect of the Person

Jean-Manuel Larralde

\section{OpenEdition}

Journals

Édition électronique

URL : https://journals.openedition.org/crdf/557

DOI : $10.4000 /$ crdf. 557

ISSN : 2264-1246

Éditeur

Presses universitaires de Caen

Édition imprimée

Date de publication : 1 novembre 2017

Pagination : 97-106

ISBN : 978-2-84133-858-0

ISSN : $1634-8842$

Référence électronique

Jean-Manuel Larralde, « Le corps des personnes détenues : de l'objet de punition au respect de la personne », Cahiers de la recherche sur les droits fondamentaux [En ligne], 15 | 2017, mis en ligne le 01 octobre 2019, consulté le 14 novembre 2022. URL : http://journals.openedition.org/crdf/557 ; DOI : https://doi.org/10.4000/crdf.557 


\title{
Le corps des personnes détenues: de l'objet de punition au respect de la personne
}

\author{
Jean-Manuel LARRALDE \\ Professeur de droit public à l'université de Caen Normandie \\ Centre de recherche sur les droits fondamentaux et les évolutions du droit (CRDFED, EA 2132)
}

I. Du « corps machine » au détenu travailleur

A. Le corps du détenu, instrument de peine

B. Le travail en prison, instrument de resocialisation

II. La prise en charge adéquate de la santé du détenu
A. L'émergence progressive d'un droit à la santé
B. La mise en place de stratégies thérapeutiques globales
C. Le refus du corps sexualisé du détenu

III. La protection de l'intégrité physique et de la dignité de la personne en détention

A. La limitation de l'usage de la force en détention

B. L'importance du principe de dignité en prison

Il a toujours existé un lien intime entre la politique pénale (ou criminelle pour reprendre le vocabulaire plus ancien...) et le corps des détenus: l'historique de la procédure et de la sanction pénales démontre bien la recherche d'une peine devant être avant tout afflictive et humiliante et constituant un châtiment exemplaire, afin de permettre l'intimidation ${ }^{1}$. Le "spectacle pénal» (pour reprendre l'expression de Jean-Marie Carbasse) a généré des peines exemplaires, destinées le plus souvent à punir le criminel "par où il avait pêché » ${ }^{2}$. La suprématie de la sanction intimidatrice conduit notamment, dès le XIII siècle, à la multiplication des peines corporelles qui se substituent aux amendes, jugées trop peu dissuasives. D'où des supplices destinés à être particulièrement explicites aux yeux de la population qui serait tentée de suivre l'exemple criminel ${ }^{3}$. La «cicatrice pénale» sous l'Ancien

1. Avant même la peine, l'obtention des preuves par l'«aveu » était souvent liée à l'infliction de supplices variés que l'on qualifierait aujourd'hui de tortures... Voir G. Pandelon, La question de l'aveu en matière pénale, thèse de doctorat en droit privé et en sciences criminelles, université d'AixMarseille, 2012. Voir également M. Foucault, Surveiller et punir: naissance de la prison, Paris, Gallimard (Bibliothèque des histoires), 1975, p. 39 sq. 2. Avec une symbolique explicite en ce qui concerne la manière dont la mort était donnée. Ainsi, les sorciers étaient livrés au bûcher, anticipation des flammes de l'enfer, les faux monnayeurs bouillis dans un chaudron, opération rappelant la fonte des métaux, etc. Voir J.-M. Carbasse, Introduction historique au droit pénal, Paris, PUF (Droit fondamental. Droit pénal), 199o, p. 222. Voir également M. Foucault, Surveiller et punir..., spéc. p. 55 sq.

3. Cette conception explique la présence, dans la liste des peines, de l'exposition des condamnés, du gibet, ou encore des exécutions par effigie (que l'on trouve, notamment, dans l'Ordonnance criminelle française de 1670). Selon un ancien ouvrage français de procédure criminelle, les exécutions 
Régime (fleur de lys ou lettres...) constitue également l'une des techniques permettant ces supplices des corps ${ }^{4}$.

La généralisation de la prison comme sanction pénale à partir du XVIII ${ }^{\mathrm{e}}$ siècle n'a pas rompu ce lien au corps. En parcourant le sommaire du magistral ouvrage de Michel Foucault Surveiller et punir 5 , on s'aperçoit immédiatement à quel point la privation de liberté carcérale est intimement liée au corps de celui qu'on enferme. Parmi les titres des chapitres, on trouve en effet «Le corps des condamnés " ${ }^{6}$, "Les moyens du bon dressement ${ }^{7}$, ou encore «Les corps dociles $»^{8}$. Pour Foucault en effet, ces «institutions complètes» que sont les prisons prennent en charge l'individu privé de sa liberté de manière totale, puisque l'institution carcérale vise à fabriquer «des corps à la fois dociles et capables ${ }^{9}$. Même à l'abri des regards de la société, le corps du détenu est partie intégrante de sa peine ${ }^{10}$. Les exécrables conditions de détention des prisons de l'Ancien Régime décrites par de nombreux commentateurs ${ }^{11}$ entraînent des souffrances dans la chair même des personnes privées de liberté. Celles-ci sont par ailleurs souvent contraintes d'utiliser leur corps comme un outil de travail, car de nombreuses prisons sont également des lieux où le travail - qu'il soit productif ou non - est une composante de la peine.

La fin du XIX ${ }^{\mathrm{e}}$ siècle et le $\mathrm{XX}^{\mathrm{e}}$ siècle connaissent une modification radicale des liens entre la prison et le corps des personnes détenues, en transformant l'institution carcérale sous l'influence de plusieurs théories dont celle de la défense sociale nouvelle ${ }^{12}$. Mettant en avant la resocialisation du délinquant, la prison contemporaine possède en effet un objectif avoué de transformation de l'individu sanctionné, à l'aide de plusieurs éléments qui forment ce que l'on appelle le traitement pénitentiaire ${ }^{13}$. En ce sens, l'Ensemble de principes pour la protection de toutes les personnes soumises à une forme quelconque de détention ou d'emprisonnement, adopté par les Nations unies en $1988^{14}$, énonce dès son premier principe que « $[t]$ oute personne soumise à une forme quelconque de détention ou d'emprisonnement est traitée avec humanité et avec le respect de la dignité inhérente à la personne humaine». De même, la lecture des Règles pénitentiaires européennes de 2006 (RPE) ${ }^{15}$ nous apprend: "Chaque détention est gérée de manière à faciliter la réintégration dans la société libre des personnes privées de liberté» (règle $n^{\circ} 6$ ), ce qui passe notamment par «des conditions de détention qui ne portent pas atteinte à la dignité humaine et offr[ent] des occupations constructives et une prise en charge permettant la préparation à leur réinsertion dans la société » (préambule). L'intégration de ce principe de dignité dans le domaine pénitentiaire a permis d'abandonner la conception qui assimilait les personnes privées de leur liberté à des corpore vili ${ }^{16}$. Bien au contraire, la "prise en charge des corps» de ces personnes par les institutions pénitentiaires, évoquée par Michel Foucault, nécessite aujourd'hui une protection physique des individus. À cet égard, c'est sans nul doute la Cour européenne qui nous offre l'approche la plus claire et explicite en la matière. L'arrêt Kudla c. Pologne a en effet posé comme principe essentiel que l'État est tenu

devaient se faire «de plein jour, sur des places publiques, attendu que la punition des criminels sert de consolation aux bons et de terreur aux méchants» (Style et manière de procéder en matière criminelle au pays de Liège, Liège - Hervé, H.-J. Urban, 1779, p. 79; cité par L. E. Alkin, «La cruauté des supplices sous l'Ancien Régime», Revue historique de droit français et étranger, 1937, p. 134). La même logique est retenue par Guillaume le Conquérant qui, dès le XI siècle, fait remplacer la peine de mort, jugée trop peu «spectaculaire», par la double mutilation de l'aveuglement et de l'émasculation. Michel Foucault évoque de son côté «le corps supplicié, dépecé, amputé, symboliquement marqué au visage ou à l'épaule, exposé vif ou mort, donné en spectacle» (M. Foucault, Surveiller et punir..., p. 14).

4. Le Code pénal de 1810 rétablira même la marque au fer rouge, infligée après le carcan, sur l'épaule droite de la victime. Voir A. Corbin, «Douleurs, souffrances et misères du corps", in Histoire du corps, A. Corbin (dir.), Paris, Seuil, 2005, vol. II, p. 236 et 240.

5. De manière tout à fait symbolique, l'ouvrage de Foucault s'ouvre sur la description minutieuse et horrifique du supplice de Damiens le 2 mars 1757 , condamné à «faire amende honorable devant la principale porte de l'Église de Paris » (Surveiller et punir..., p. 9-11).

6. M. Foucault, Surveiller et punir..., p. 9.

7. Ibid., p. 172.

8. Ibid., p. 137

9. Ibid., p. 301.

10. «[...] même si [les systèmes punitifs] ne font pas appel à des châtiments violents ou sanglants, même lorsqu'ils utilisent les méthodes "douces" qui enferment ou corrigent, c'est bien toujours du corps qu'il s'agit - du corps et de ses forces, de leur utilité et de leur docilité, de leur répartition et de leur soumission» (ibid., p. 30). Il ajoute par ailleurs: "Que les punitions en général et que la prison relèvent d'une technologie politique du corps, c'est peut-être moins l'histoire qui me l'a enseigné que le présent» (ibid., p. 35).

11. Parmi lesquels John Howard qui, en 1777, publie un ouvrage au retentissement considérable, The State of the Prisons [L'état des prisons], où il dénonce une désorganisation complète des conditions de détention, leur aspect barbare et malsain.

12. Dont les plus célèbres promoteurs sont le Belge Adolphe Prins (La défense sociale et les transformations du droit pénal, Bruxelles - Leipzig, Misch et Thron, 1910) et le Français Marc Ancel (La défense sociale nouvelle: un mouvement de politique criminelle humaniste, Paris, Cujas, 1954).

13. Le Congrès pénitentiaire de Bruxelles en 1847 évoque comme fonction essentielle de la sanction pénale la transformation du comportement de l'individu: «L'amendement du condamné comme but principal de la peine est un principe sacré dont l'apparition formelle dans le domaine de la science et surtout dans celui de la législation est toute récente». En France de tels objectifs seront définis dans les principes de la commission Amor de 1945: « $1^{\circ}$. La peine privative de liberté a pour but essentiel l'amendement et le reclassement du condamné»; " $3^{\circ}$. Le traitement infligé aux prisonniers hors de toute promiscuité corruptive doit être humain, exempt de vexations, et tendre principalement à son instruction générale et professionnelle et à son amélioration» (cité par M. Foucault, Surveiller et punir..., p. 274).

14. Ensemble de principes pour la protection de toutes les personnes soumises à une forme quelconque de détention ou d'emprisonnement, adopté par l'Assemblée générale des Nations unies dans sa résolution 43/173 du 9 décembre 1988.

15. Recommandation Rec (2006) 2 du Comité des ministres aux États membres sur les Règles pénitentiaires européennes adoptée par le Comité des ministres du Conseil de l'Europe le 11 janvier 2006 lors de la $952^{\mathrm{e}}$ réunion des délégués des ministres.

16. Lire sur ce point G. Chamayou, Les corps vils : éthique et politique de l'expérimentation humaine aux XVII et XVIII ${ }^{e}$ siècles, Paris, Les empêcheurs de penser en rond - La Découverte, 2008. 
[...] de s'assurer que tout prisonnier est détenu dans des conditions qui sont compatibles avec le respect de la dignité humaine, que les modalités d'exécution de la mesure ne soumettent pas l'intéressé à une détresse ou à une épreuve d'une intensité qui excède le niveau inévitable de souffrance inhérent à la détention et que, eu égard aux exigences pratiques de l'emprisonnement, la santé et le bien-être du prisonnier sont assurés de manière adéquate, notamment par l'administration des soins médicaux requis ${ }^{17}$.

Euvrant pour une prison plus humaine, la Cour de Strasbourg rappelle constamment que la mission de la prison n'est plus aujourd'hui le «dressement» des siècles passés, mais le «traitement pénitentiaire», qui nécessite de limiter voire d'interdire certaines pratiques ou mesures en détention. Ces idées, qui se sont également imposées en droit interne ${ }^{18}$, ont entraîné une triple transformation du rapport entre le corps des détenus et la prison, en aboutissant progressivement à transformer le «corps machine» en prison en détenu travailleur (I), en exigeant une prise en charge adéquate de la santé du détenu (II) et en protégeant enfin l'intégrité physique et la dignité de la personne en détention (III).

\section{Du « corps machine" au détenu travailleur}

Si Michel Foucault a pu développer la figure du « détenumachine ${ }^{19}$, dont le corps constitue l'un des éléments de la peine (A), l'époque contemporaine est au contraire celle de la recherche d'un travail instrument de resocialisation, qui peine toutefois à reconnaître au détenu travailleur l'ensemble des droits sociaux dont il devrait légitimement disposer (B).

\section{A. Le corps du détenu, instrument de peine}

L'histoire du travail pénitentiaire se confond avec l'histoire de la sanction pénale, puis de la prison elle-même. Si, à Rome, les détenus étaient envoyés ad metalla (aux mines) ou opus publicum (aux travaux publics), l'Ancien Régime inventera la forme la plus aboutie, celle des galères $^{20}$. Il s'agissait alors d'utiliser et d'exploiter le travail du détenu astreint, parfois même jusqu'à l'épuisement. Les premières véritables prisons apparues au $\mathrm{XVI}^{\mathrm{e}}$ siècle revêtaient ainsi le caractère d'établissements de travail pour les vagabonds et les mendiants où les tâches imposées sont l'un des éléments afflictifs de la peine. En 1557, à Londres, l'ancien palais désaffecté de Bridwell devient un atelier de travail obligatoire et éducatif pour les vagabonds, en présentant le double caractère de prison et d'établissement de sûreté. Sur ce modèle, l'Angleterre fondera d'autres maisons de travail, appelées bridwells. En 1596, s'ouvre à Amsterdam le Rasphuis, institution destinée aux jeunes délinquants. Le but de cette prison, créée de toutes pièces, est de transformer et d'amender les détenus par le travail et l'éducation religieuse, l'essentiel du régime étant constitué par un travail de tissage (qui sera, par la suite, remplacé par le râpage du bois de campêche $)^{21}$. L'application ultime de cette conception consistait à imposer des travaux inutiles ou improductifs. L'Angleterre se révélera l'un des États les plus «inventifs» en matière de travail afflictif, avec les pratiques du shot-drill (le détenu devant parcourir une vaste salle de long en large en portant un boulet de canon), de l'écureuil ou du crank (le détenu devant tourner dans un cylindre ou actionner une manivelle, le plus souvent dans le seul but d'actionner un compteur servant à comptabiliser le nombre d'unités de travail accomplies) ${ }^{22}$. Le travail exigé des détenus sera ainsi pendant longtemps afflictif, voire imposé de manière publique et dégradante. Ainsi, l'article 1o du Code pénal français de 1810 prévoyait encore que les condamnés aux travaux forcés devaient être employés aux travaux les plus pénibles, traîner à leurs pieds un boulet ou être attachés deux à deux à une chaîne, lorsque la nature du travail le permettait. Le corps du détenu est alors réduit à un instrument astreint à des tâches imposées, le travail physique étant une composante de la peine ${ }^{23}$.

Transformant radicalement l'approche théorique de la prison, les théories pénologiques qui vont se multiplier à partir de la fin du XIX ${ }^{\mathrm{e}}$ siècle vont aboutir à modifier le rôle du travail; on passe alors du détenu-machine au détenu-travailleur, qui doit normalement trouver dans l'activité professionnelle l'un des leviers de sa resocialisation.

17. Cour EDH, 26 octobre 200o, Kudla c. Pologne, nº $30210 / 96, \$ 94$.

18. Selon l'article $1^{\text {er }}$ de la loi pénitentiaire 2009-1436 du 24 novembre 2009: «Le régime d'exécution de la peine de privation de liberté concilie la protection de la société, la sanction du condamné et les intérêts de la victime avec la nécessité de préparer l'insertion ou la réinsertion de la personne détenue afin de lui permettre de mener une vie responsable et de prévenir la commission de nouvelles infractions».

19. «Le travail devait être la religion des prisons. À une société-machine, il fallait des moyens de réforme purement mécaniques. Fabrication d'individus-machines mais aussi de prolétaires» (M. Foucault, Surveiller et punir..., p. 246).

20. Voir, inter alia, M. Foucault, Surveiller et punir..., p. 261 sq.

21. Voir, à ce sujet, H.-J. Kerner, "Les sanctions pénales classiques et leurs altérations dans les politiques criminelles européennes", Annales internationales de criminologie, vol. 25, $\mathrm{n}^{\circ} 1$ et 2, 1987, p. 92. Voir également M. Foucault, Surveiller et punir..., p. 123 sq.

22. Voir G. Kellens, La mesure de la peine: précis de pénologie et de droit des sanctions pénales, Liège, Faculté de droit, d'économie et de sciences sociales, 1982, p. 110 ; R. S. E. Hinde, The British Penal System 1773-1950, Londres, G. Duckworth, 1951, p. 155 sq.

23. «La prison n'est pas un atelier; elle est, il faut qu'elle soit en elle-même une machine dont les détenus-ouvriers sont à la fois les rouages et les produits; elle les "occupe" et cela "continuellement fût-ce dans l'unique but de remplir leurs moments [...]" "; "Le travail devait être la religion des prisons» (M. Foucault, Surveiller et punir..., p. 245 et 246). 


\section{B. Le travail en prison, instrument de resocialisation}

À ces approches faisant de la personne du condamné un «détenu-machine ${ }^{24}$ se substituera progressivement l'idée d'un travail élément constitutif de la peine, à la fois puissant levier d'amélioration du détenu et soubassement du système disciplinaire des établissements. Le travail en détention ne doit pas alors être autre chose que la continuation du travail en milieu libre. L'expression la plus claire de cette théorie se trouve formulée par l'article 60.1 de l'Ensemble de règles minima pour le traitement des détenus élaboré par le Conseil économique et social des Nations unies en $1955^{25}$ :

Le régime de l'établissement doit chercher à réduire les différences qui peuvent exister entre la vie en prison et la vie libre dans la mesure où ces différences tendent à affaiblir le sens de la responsabilité du détenu ou le respect de la dignité de la personne ${ }^{26}$.

La règle 74.1 ajoute: «Les précautions prescrites pour protéger la sécurité et la santé des travailleurs libres doivent également être prises dans les établissements pénitentiaires ». Plus près de nous, la règle 26.1 des Règles pénitentiaires européennes de 2006 va plus loin encore en prévoyant que: «Le travail en prison doit être considéré comme un élément positif du régime carcéral et en aucun cas être imposé comme une punition ». Certes, la prison moderne n'est pas celle d'un véritable détenu travailleur ${ }^{27}$, même si le Code de procédure pénale prévoit que « [1]es dispositions nécessaires doivent être prises pour qu'un travail productif et suffisant pour occuper la durée normale d'une journée de travail soit fourni aux personnes détenues» (art. D. 432-2) et que " [1]'organisation, les méthodes et les rémunérations du travail doivent se rapprocher autant que possible de celles des activités professionnelles extérieures afin notamment de préparer les détenus aux conditions normales du travail libre» (art. D. 433). Souvent répétitif et limité à des tâches simples ${ }^{28}$, mal rémunéré, et en dehors des règles de droit commun régissant le contrat de travail et son exécution ${ }^{29}$, le travail en détention n'est cependant plus assimilable $\mathrm{au}$ «corps assujetti» décrit par Michel Foucault ${ }^{30}$, lié à la recherche d'une souffrance physique inhérente à la privation de liberté carcérale.

Si la prison moderne marque le rejet du «détenumachine», elle marque également, sous l'influence identique des théories du traitement pénitentiaire, la reconnaissance du «détenu-patient », qui nécessite une prise en charge adéquate de sa santé.

\section{La prise en charge adéquate de la santé du détenu}

L'évolution de la prise en compte de la situation sanitaire des détenus est elle aussi intimement liée à l'évolution des conceptions pénologiques, qui vont imposer l'idée d'un véritable droit à la santé pour les personnes incarcérées $(\mathrm{A})$, ce qui exige la mise en place de stratégies thérapeutiques globales $(\mathrm{B})$. Ces indéniables progrès tardent toutefois à pleinement reconnaître le corps sexualisé du détenu, dont la protection de l'intimité sexuelle reste encore partielle $(\mathrm{C})$.

\section{A. L'émergence progressive d'un droit à la santé}

Il est inexact de dire que la prise en charge de la santé des détenus n'a pas constitué, au moins d'un point de vue théorique, une préoccupation ancienne. Dès 1603, Bouteiller dans sa Somme rurale indique ainsi que «la prison ne doit pas être griève en sorte qu'elle puisse ou doive empirer le corps du prisonnier», exigence qui pénétrera

24. «À une société-machine, il fallait des moyens de réforme purement mécaniques. Fabrication d'individus-machines mais aussi de prolétaires » (M. Foucault, Surveiller et punir..., p. 246).

25. Ensemble de règles minima pour le traitement des détenus, adopté par le premier Congrès des Nations unies pour la prévention du crime et le traitement des délinquants, tenu à Genève en 1955 et approuvé par le Conseil économique et social dans ses résolutions $663 \mathrm{C}$ (XXIV) du 31 juillet 1957 et 2076 (LXII) du 13 mai 1977.

26. Disposition à laquelle fait écho la règle 26.7 des Règles pénitentiaires européennes de 2006, selon laquelle: «L'organisation et les méthodes de travail dans les prisons doivent se rapprocher autant que possible de celles régissant un travail analogue hors de la prison, afin de préparer les détenus aux conditions de la vie professionnelle normale».

27. Comme l'a démontré le récent arrêt de la Cour européenne des droits de l'homme Meier c. Suisse du 9 février 2016 (nº 10109/14), dans lequel la Cour européenne des droits de l'homme valide l'obligation de travailler imposée à un détenu ayant atteint l'âge de la retraite. Voir N. Ferran, Dedans-dehors, n 91, avril 2016, p. 47. De même, l'arrêt Stummer c. Autriche du 7 juillet 2011 ( ${ }^{\circ}$ 37452/o2, $\left.\$ 110\right)$ ne voit pas dans le refus d'affilier les détenus qui travaillent en prison au régime d'assurance-retraite une violation de la Convention européenne des droits de l'homme (Convention EDH). Il ne faut pas non plus oublier que si l'article 4 de la Convention EDH prohibe le travail forcé, son $\$ 3 . a$ ajoute aussitôt que «N'est pas considéré comme "travail forcé ou obligatoire” au sens du présent article: tout travail requis normalement d'une personne soumise à la détention dans les conditions prévues par l'article 5 de la présente Convention, ou durant sa mise en liberté conditionnelle».

28. Voir Dedans-Dehors, ${ }^{\circ}$ 55, mai-juin 2006, Travail des détenus à bout de souffle, p. 14.

29. Amené à se prononcer sur la question de la nature de l'acte juridique sur lequel reposent les activités professionnelles des personnes détenues, le Conseil constitutionnel, dans sa décision n 2015-485 QPC du 25 septembre 2015, a jugé que l' «acte d'engagement» (document signé par le chef d'établissement et la personne détenue, qui énonce les droits et obligations professionnels de celle-ci ainsi que ses conditions de travail et sa rémunération) ne viole ni la liberté contractuelle, ni le principe de dignité des personnes. Voir le communiqué de presse très critique du Contrôleur général des lieux de privation de liberté rendu public après cette décision du Conseil, en ligne: http://www.cglpl.fr/wp-content/ uploads/2013/o6/Com-de-presse_CGLPL_QPC-travail.pdf.

30. M. Foucault, Surveiller et punir..., p. 31 
le droit positif avec l'Ordonnance criminelle française de $1670^{31}$. En Angleterre, c'est dès 1774 que la Chambre des communes adopte une loi visant à préserver la santé des prisonniers ${ }^{32}$. Plus près de nous, c'est la douche qui sera inventée en prison, à Bonne-Nouvelle à Rouen, afin de permettre, selon son promoteur, de réaliser de «sérieux progrès dans l'hygiène des populations agglomérées, prisons, casernes, internats, etc. ${ }^{33}$. Mais les conditions matérielles de détention fortement dégradées ont rendu l'ensemble de ces louables objectifs sanitaires pour le moins virtuels ${ }^{34}$.

Ce sont ici encore les nouvelles théories pénales et pénologiques apparues pour l'essentiel au $\mathrm{XX}^{\mathrm{e}}$ siècle qui vont offrir une nouvelle perspective en la matière. En 1990, les Nations unies dans leurs Principes fondamentaux relatifs au traitement des détenus précisent qu'ils «ont accès aux services de santé existant dans le pays, sans discrimination aucune du fait de leur statut juridique $»^{35}$. Les Règles pénitentiaires européennes de 2006 vont plus loin encore en la matière, en indiquant que

Les locaux de détention [...] doivent satisfaire aux exigences de respect de la dignité humaine et, dans la mesure du possible, de la vie privée, et répondre aux conditions minimales requises en matière de santé et d'hygiène [...]. (règle 18.1)

Ce texte pose surtout le principe de services de santé pénitentiaire fonctionnant sur la base des standards de la société libre, puisque «[l]a politique sanitaire dans les prisons doit être intégrée à la politique nationale de santé publique et compatible avec cette dernière» (règle 40.2) ${ }^{36}$.

Ces exigences, qui ont été intégrées au droit français dès $1994^{37}$, ont été précisées par une désormais abondante jurisprudence de la Cour européenne des droits de l'homme, qui depuis l'arrêt Kudla fait de «la santé et $[\mathrm{du}]$ bien-être du prisonnier [...] assurés de manière adéquate, notamment par l'administration des soins médicaux requis ", des composantes d'une privation de liberté pénitentiaire respectueuse de la dignité humaine ${ }^{38}$. Depuis cette décision fondatrice, la Cour de Strasbourg a eu l'occasion de préciser ses exigences, notamment dans son arrêt Mouisel c. France du 14 novembre 2002 qui pose ici un double principe général. D’une part, elle rappelle qu'il n'existe pas en tant que telle une obligation générale de libérer un détenu pour motifs de santé. Mais, d'autre part,

[...] la santé d'une personne privée de liberté fait désormais partie des facteurs à prendre en compte dans les modalités de l'exécution de la peine privative de liberté, notamment en ce qui concerne la durée du maintien en détention ${ }^{39}$.

\section{B. La mise en place de stratégies thérapeutiques globales}

L'application effective des exigences de l'arrêt Kudla est claire: un suivi médical doit être proposé à tout détenu,

31. Selon l'article 21 de l'Ordonnance criminelle française de 1670: «[...] les guichetiers visiteront tous les jours les prisonniers dans les cachots, et devront indiquer ceux qui sont malades, pour qu'ils soient visités par les médecins et au besoin transférés dans des chambres» (A. Esmein, Histoire de la procédure criminelle en France, Paris, L. Larose et Forcel, 1882, p. 228). Il faut toutefois attendre 1780 pour qu'une autre ordonnance prévoie la création d'infirmeries dans les prisons.

32. Loi dont le contenu sera décrit par John Howard en ces termes: elle «exige qu'un chirurgien ou un pharmacien soit nommé dans chaque établissement. Sa tâche est, en premier lieu, d'ordonner le transfert immédiat des malades vers l'infirmerie; et contrôler qu'ils ont un régime et une literie correcte. Leurs fers doivent être enlevés; et ils doivent avoir, non seulement des médicaments, mais également un régime alimentaire approprié à leur condition. Il doit les visiter quotidiennement et avec diligence; ne pas les laisser à des assistants. Il doit constamment inculquer la nécessité de la propreté et de l'air frais; et le danger d'entasser les prisonniers: et il doit recommander ce qu'il ne peut imposer» (J. Howard, L'état des prisons; cité par H. K. Snell, «The Prison Medical Service», The Howard Journal, vol. 10, n 2, 1959, p. 75; nous traduisons).

33. Selon les mots du docteur Merry Delabost, médecin-chef à la prison de Bonne-Nouvelle, cité par H. Dajon, «La douche, une invention d'un médecin des prisons, le docteur Merry Delabost», Criminocorpus, 26 janvier 2013, en ligne: http://criminocorpus.revues.org/2006.

34. Sans parler d'autres pratiques telles que l'utilisation de détenus à des fins d'expérimentations médicales: en 1772, les prisonniers de la prison de Newgate en Angleterre se portèrent «volontaires» pour se faire inoculer le virus de la variole plutôt qu'être pendus. Tous survivants, ils furent donc relâchés. De même, Hernandez inocula la syphilis à des détenus du bagne de Toulon en 1812. Ces expérimentations ne cesseront pas au $\mathrm{XX}^{\mathrm{e}}$ siècle avec les recherches sur le béribéri menées par Strong et Crowell sur vingt-huit prisonniers (en échange de quelques paquets de cigarettes!) et qui aboutiront à la mort d'un des détenus, ou encore, en 1916, les expériences concernant le rôle d'un régime carencé sur la pellagre menées par Goldberger et Wheeler sur onze prisonniers d'un pénitencier de l'État du Missouri. Pour l'ensemble de ces expérimentations menées sur les personnes privées de liberté, voir R. Franchitti, L'expérimentation humaine dans l'histoire de la médecine, thèse de doctorat, université Paris 13 , 1981.

35. Principes fondamentaux relatifs au traitement des détenus, adoptés par l'Assemblée générale des Nations unies dans sa résolution $45 / 111$ du 14 décembre 1990, $\$ 9$

36. Voir également les règles 40.1: «Les services médicaux administrés en prison doivent être organisés en relation étroite avec l'administration générale du service de santé de la collectivité locale ou de l'État» et 40.5: «[...] chaque détenu doit bénéficier des soins médicaux, chirurgicaux et psychiatriques requis, $y$ compris ceux disponibles en milieu libre».

37. La loi no 94-43 du 18 janvier 1994 et le décret n ${ }^{\circ}$ 94-929 du 27 octobre 1994 confient au service public hospitalier l'organisation des soins dispensés aux détenus. Le préfet de région doit désigner pour chaque établissement pénitentiaire un établissement public de santé, chargé de dispenser les soins aux détenus et de participer à l'accueil et aux urgences. Les détenus peuvent donc, en fonction de leur état, soit être conduits en consultation spécialisée à l'hôpital dont dépend leur établissement, soit recevoir la visite, au sein de celui-ci, de médecins vacataires. L'article 46 de la loi pénitentiaire 2009-1436 du 24 novembre 2009 ajoute par ailleurs que: «La prise en charge de la santé des personnes détenues est assurée par le service public hospitalier dans les conditions prévues par le code de la santé publique».

38. Cour EDH, 26 octobre 200o, Kudla c. Pologne, $\$ 94$

39. Cour EDH, 14 novembre 2002, Mouisel c. France, $\mathrm{n}^{\circ}$ 67263/01, $\$ 43$ et 45 : Recueil Dalloz, 2003, p. 303, note H. Moutouh; J.-P. Céré, «Le maintien en détention de malades graves constitue un traitement inhumain et dégradant ", Revue trimestrielle des droits de l'homme, $\mathrm{n}^{\circ} 55,2003, \mathrm{p} .1007$ sq. H. Tigroudja, «Le maintien en détention d'une personne atteinte d'un cancer au regard de l'article 3 de la Convention européenne des droits de l'homme», Petites affiches, $\mathrm{n}^{\circ}$ 122, 19 juin 2003, p. 18 sq. 
et des soins médicaux appropriés doivent être dispensés à des détenus malades. La Cour s'autorise désormais à vérifier la nature du traitement médical administré. Et il est aujourd'hui acquis que cette protection de la santé ne se limite pas à la lutte contre des symptômes, mais relève d'une stratégie thérapeutique globale, qui doit être adéquate et rapide ${ }^{40}$. Cette exigence de protection des corps incluse dans l'article 3 de la Convention EDH va désormais très loin, puisque la Cour exige par exemple qu'au nom du droit à la protection de la santé de chaque individu, un détenu non fumeur soit incarcéré dans une cellule occupée uniquement par des codétenus non fumeurs ${ }^{41}$, qu'un prisonnier puisse se voir prescrire rapidement une prothèse dentaire (ainsi qu'un régime alimentaire adéquat!) ${ }^{42}$, ou encore qu'un prisonnier ayant des difficultés à marcher se voie attribuer une canne ${ }^{43}$.

Pèsent enfin sur les États des exigences renforcées pour certaines catégories de détenus nécessitant des modalités de prise en charge particulières. Ainsi en est-il des personnes lourdement handicapées qui doivent bénéficier de conditions de détention adaptées à leurs besoins spécifiques ${ }^{44}$. Cette prise en charge des corps aboutit également à une prise en compte particulière des personnes âgées en détention ${ }^{45}$. Bien évidemment, cette volonté de protéger la santé des détenus s'insère dans la politique prétorienne des juges strasbourgeois visant à imposer aux autorités pénitentiaires nationales des conditions matérielles de détention dignes et humaines, jurisprudence initiée pour les détenus malades par l'arrêt Kalachnikov c. Russie du 15 juillet $2002^{46}$. Comme la Cour l'a récemment rappelé dans son arrêt Martzaklis et autres c. Grèce du 9 juillet 2015, un détenu malade (en l'espèce séropositif) doit «être placé dans un endroit en adéquation avec ses besoins médicaux et son bien-être ${ }^{47}$.

\section{Le refus du corps sexualisé du détenu}

Seul le respect de la sexualité du détenu constitue encore une question prise en compte de manière peu satisfaisante. Si l'on définit la santé, comme le fait l'Organisation mondiale de la santé (OMS), comme « un état complet de bien-être physique, mental et social, et ne consist[ant] pas seulement en une absence de maladie ou d'infirmité ${ }^{48}$, nul ne peut contester que le respect de la sexualité des personnes incarcérées devrait en constituer l'une des composantes. Or, comme l'a notamment relevé le Conseil de l'Europe,

La prison signifie également la mise en suspens de l'épanouissement affectif et sexuel du couple, qu'il soit hétéro ou homosexuel. La prison n'est pas un lieu de tendresse. La privation de cette dimension humaine est une conséquence indirecte et cruciale de la peine privative de liberté ${ }^{49}$.

La sexualité des personnes privées de liberté a en effet constitué pendant fort longtemps un sujet totalement occulté, renvoyant à l'idée que la privation de sexualité constituait l'un des éléments inhérents de toute incarcération. Il est d'ailleurs tout à fait frappant de remarquer que les si détaillées Règles pénitentiaires européennes n'ont jamais évoqué le respect de la sexualité de la personne

40. Dans son arrêt Veretco c. République de Moldova du 7 avril 2015 ( $n^{\circ}$ 679/13) relatif à un détenu souffrant de fractures des côtes et d'une pneumonie, la Cour exige que les autorités pénitentiaires prodiguent avec diligence les soins adéquats aux détenus malades. Ainsi, se contenter de faire examiner un détenu souffrant d'une grave maladie cérébrale, qui entraîne des symptômes incluant des maux de tête sévères, des crises d'épilepsie, des nausées et des insomnies, par un auxiliaire médical et un psychiatre de la prison alors qu'il nécessitait une intervention neurochirurgicale viole l'article 3 de la Convention EDH (arrêt Budanov c. Russie, 9 janvier 2014, no 66583/11). Voir aussi Kolesnikovich c. Russie et Litvinov c. Russie du 22 mars 2016 ( $n^{\circ} 44694 / 13$ et 32863/13).

41. Pour un détenu asthmatique, confiné vingt-trois heures par jour dans sa cellule: Cour EDH, 13 septembre 2005, Ostrovar c. Moldavie, n $35207 / 03$ : L'actualité juridique. Droit pénal, 2005, p. 421, obs. J.-P. Céré.

42. Cour EDH, 2 février 2016, Drăgan c. Roumanie, nº 65158/o9.

43. Cour EDH, 9 juin 2016, Mekras c. Grèce, n $12863 / 14$.

44. Cour EDH, 10 juillet 2001, Price c. Royaume-Uni, $\mathrm{n}^{\circ}$ 33394/96. La Cour, sans relever une volonté manifeste d'avilissement ou d'humiliation de la requérante (handicapée des quatre membres), juge que sa détention dans des conditions où elle souffre sérieusement du froid et risque d'avoir des douleurs en raison de la dureté et de l'inaccessibilité de son lit, qu'elle ne peut que très difficilement quitter pour aller aux toilettes, ou se laver, constitue un traitement dégradant au sens de l'article 3. Voir F. Sudre, «Droit de la Convention européenne des droits de l'homme, l'intégrité physique et morale de la personne (article 3)", La semaine juridique, édition générale, $\mathrm{n}^{\circ}$ 3, 2002, p. 128 sq.

45. Dans l'affaire Hénaf c. France du 27 novembre 2003 (n $65436 / 01)$, la Cour estime qu'en raison de différents éléments, dont l'âge du requérant (75 ans à l'époque des faits), l'usage d'entraves lors d'un transfert et lors d'une hospitalisation constitue une mesure disproportionnée au regard des nécessités de la sécurité (et ce d'autant que deux policiers avaient été spécialement placés en faction devant la chambre du requérant). Dans le même sens, dans un arrêt Farbtuhs c. Lituanie du 2 décembre 2004 ( $\mathrm{n}^{\circ}$ 4672/02), la Cour juge que le maintien en détention pendant un an et neuf mois d'un détenu (condamné pour crime contre l'humanité et génocide) âgé de 84 ans, paraplégique, invalide et atteint de maladies graves et incurables, n'était pas adéquat en raison de son âge, de son infirmité et de son état de santé et créait chez l'intéressé des «sentiments constants d'angoisse, d'infériorité et d'humiliation suffisamment forts pour constituer un traitement dégradant au sens de l'art. 3 de la Convention » ( $\$ 61$ ). Voir également l'arrêt Helhal c. France du 19 février 2015 (n 10401/12).

46. Cour EDH, 15 juillet 2002, Kalachnikov c. Russie, $\mathrm{n}^{\circ}$ 47095/99. Voir la fiche thématique de la Cour EDH, «Conditions de détention et traitement des détenus", avril 2017, en ligne: http://www.echr.coe.int/Documents/FS_Detention_conditions_FRA.pdf.

47. Cour EDH, 9 juillet 2015, Martzaklis et autres $c$. Grèce, $\mathrm{n}^{\circ}$ 20378/13, $\$ 71$. En l'espèce, le transfert des intéressés à l'hôpital pénitentiaire Aghios Pavlos a conduit les détenus à être à proximité de détenus souffrant de maladies telles que la tuberculose ou l'hépatite et les a donc exposés «à une souffrance physique et mentale allant au-delà de celle inhérente à la détention ", constitutive d'une violation de l'article 3 de la Convention ( $\$ 75$ ).

48. OMS, Constitution adoptée par la Conférence internationale de la santé, tenue à New York du 19 juin au 22 juillet 1946, signée par les représentants de 61 États le 22 juillet 1946 et entrée en vigueur le 7 avril 1948 (Actes officiels de l'Organisation mondiale de la santé, $\mathrm{n}^{\circ}$ 2, p. 100).

49. Earl of Dundee, Effets de la détention sur le plan familial et social, Assemblée parlementaire du Conseil de l'Europe, rapport $n^{\circ} 7816,15$ mai 1997, p. 17. Voir également A. Monnereau, «La privation sexuelle et affective du prisonnier», Promovere, n 38, 1984, p. 71-77; E. Swinnen, «La sexualité en prison ", Bulletin de l'administration pénitentiaire, 1981, p. 259-282 et 1982, p. 15-40, 129-150 et 189-201. 
incarcérée ${ }^{50}$. La jurisprudence européenne s'est également toujours refusée à reconnaître un droit à la sexualité pour les personnes incarcérées ${ }^{51}$. Au plan interne, les députés ont également eu l'occasion de dénoncer ce tabou, aboutissant à des relations sexuelles tolérées lors des parloirs, cette

[...] solution choisie procéd[ant] plus du poids de la coutume, d'une politique du directeur, que d'une véritable réflexion sur la sexualité en prison. Il faut pourtant savoir que ces relations, lorsqu'elles ont lieu, se déroulent dans des conditions indignes, avec des aménagements rudimentaires qui placent le couple, les familles présentes et leurs enfants, les surveillants, dans une situation extrêmement gênante ${ }^{52}$.

Certes, la mise en place en France de techniques telles que les parloirs familiaux ou les unités de vie familiale (UVF) ${ }^{53}$ permet de reconnaître la sexualité en prison, sans pour autant en faire un élément «normal» de la vie en détention ${ }^{54}$.

Permettant une meilleure prise en charge de la santé des détenus ${ }^{55}$, les conceptions du traitement pénitentiaire ont même permis d'aller plus loin, en cherchant à purger les prisons de comportements ou de pratiques attentatoires à la dignité humaine et portant atteinte à l'intégrité des personnes en détention.

\section{La protection de l'intégrité physique et de la dignité de la personne en détention}

Lieux fermés, devant prendre en charge des publics multiples et souvent difficiles, les prisons sont des espaces dans lesquels les primordiales exigences de sécurité et du maintien de l'ordre ont pu s'exprimer par un usage légitime de la force. Sous la pression du juge européen cette analyse est maintenant dépassée car les corps des détenus sont aujourd'hui mieux protégés par l'émergence d'un double principe, qui est celui de la limitation de l'usage de la force en détention (A), qui s'accompagne de la reconnaissance de l'application du principe de dignité à l'intérieur des établissements pénitentiaires (B).

\section{A. La limitation de l'usage de la force en détention}

Institution fermée, la prison a longtemps constitué un milieu dans lequel a existé une certaine acceptation, voire légalisation de la violence, comme le démontre l'intéressante évolution des organes du Conseil de l'Europe en la matière. Pour la Commission européenne des droits de l'homme en 1966, des mesures violentes employées contre un détenu pour le faire regagner la cellule dont il s'était échappé n'étaient ainsi pas contraires à l'article 3 de la Convention $\mathrm{EDH}^{56}$, pas plus que l'usage de la force par les gardiens, vis-à-vis d'un détenu refusant de quitter sa cellule ${ }^{57}$. Encore plus précisément, cette même Commission a pu déclarer en 1969 que certaines brutalités, qui «peuvent prendre la forme de gifles ou de coups donnés de la main sur la tête ou le visage», ne violent pas l'article 3 de la Convention EDH, car elles sont admises ou considérées comme normales par les détenus ${ }^{58}$ ! Cette jurisprudence semble aujourd'hui bien éloignée des standards actuels de la Cour européenne des droits de l'homme qui, depuis son

50. Le constat s'appliquant aux versions de 1973 (résolution (73) 5), 1987 (recommandation R (87) 3), comme 2006 (recommandation Rec (2006) 2).

51. Si la Commission européenne des droits de l'homme a pu se féliciter en 1970 « du courant réformateur qui s'est manifesté dans plusieurs pays européens vers une amélioration des conditions de détention et des possibilités des détenus de continuer à mener leur vie conjugale à l'intérieur de certaines limites" (X. c. République fédérale d'Allemagne, 4 février 1970, n 3603/68, Annuaire de la Convention européenne des droits de l'homme 1970, M. Nijhoff, La Haye, 1972, vol. XIII, p. 333 sq.), cela ne l'a pas empêchée ensuite de préciser que la défense de l'ordre pénitentiaire permet de ne pas autoriser de relations sexuelles de couples mariés en détention, et ce même si les époux demeurent dans le même établissement. En effet, «la sécurité et le bon ordre seraient sérieusement compromis si tous les détenus mariés étaient autorisés à poursuivre leur vie conjugale en prison», car «le respect de la vie privée exigerait que les autorités pénitentiaires renoncent à leur droit de surveillance constante» (X. et $Y$. c. Suisse, 3 octobre 1978, no 8166/78, Décisions et rapports, vol. 13, p. 241 sq.). De son côté, la Cour européenne des droits de l'homme a toujours refusé de consacrer un droit à la sexualité des détenus; Cour EDH, 23 février 2012, Trosin c. Ukraine, $\mathrm{n}^{\circ}$ 39758/05, \$39.

52. J. Floch, Rapport fait au nom de la commission d'enquête sur la situation dans les prisons françaises, $\mathrm{n}^{\circ} 2851$, Assemblée nationale, 28 juin 2000 , t. I. Les juridictions administratives ont d'ailleurs eu l'occasion de rappeler récemment cette tolérance de la sexualité en prison. Le tribunal administratif de Nantes a ainsi annulé une sanction de suppression de parloir sans dispositif de séparation pendant trois visites, "considérant que si M. X reconnaît avoir eu un rapport sexuel avec sa concubine [...] il est constant que personne n'a été témoin de ce rapport» (TA Nantes, 25 octobre $2007, \mathrm{n}^{\circ} 062824$ ).

53. Voir «Le maintien des liens familiaux» sur le site du ministère de la Justice: http://www.justice.gouv.fr/prison-et-reinsertion-10o36/la-vie-endetention-10039/le-maintien-des-liens-familiaux-12006.html

54. Voir Dedans-dehors, $\mathrm{n}^{\circ}$ 90, décembre 2015, Sexualité en prison: la grande hypocrisie, p. 8 sq.; A. Gaillard, Sexualité et prison: désir affectif et désirs sous contrainte, Paris, M. Milo, 2009.

55. Même si les conditions sanitaires en prison restent encore très précaires et assez éloignées des standards extérieurs. Comme le souligne le Comité consultatif national d'éthique pour les sciences de la vie et de la santé dans son avis nº 94 de novembre 2006 relatif à la médecine et à la santé en prison, «l'accès aux soins et à la protection de la santé en prison continue de poser des problèmes éthiques majeurs» (p. 2), en particulier à cause de «la surpopulation carcérale et [du] non-respect du droit à l'hygiène, à l'intimité, à la salubrité des locaux et à des conditions de vie non dégradantes pour la santé physique et mentale» (p. 18). Voir également, FARAPEJ, «La santé à l'épreuve de la prison", $20^{\text {es }}$ journées nationales prison, 2013, dossier d'animation, 28 p.; La santé en action, $\mathrm{n}^{\circ}$ 430, décembre 2014, dossier «Prison: quelle place pour la promotion de la santé?», K. Chemlal (dir.), p. 6 sq.; T. Le-Luong, préface à Promotion de la santé en milieu pénitentiaire: référentiel d'intervention, K. Chemlal, P. Echard-Bezault, P. Deutsch (dir.), Saint-Denis, INPES (Santé en action), 2014.

56. Commission EDH, 13 décembre 1966, Zeidler-Kornmann c. RFA, $\mathrm{n}^{\circ}$ 2686/65, Recueil, $\mathrm{n}^{\circ}$ 22, p. 1.

57. Commission EDH, 17 mai 1969, Wemhoff c. RFA, $\mathrm{n}^{\circ} 3448 / 67$, Recueil, $\mathrm{n}^{\circ} 30, \mathrm{p} .56$.

58. Commission EDH, 5 novembre 1969, Affaire grecque, $\mathrm{n}^{\circ} 3321 / 67$ à 3323/67, Annuaire de la Convention européenne des droits de l'homme 1969, M. Nijhoff, La Haye, 1971, vol. XII, volume spécial, chap. IV/A, p. 186 
arrêt Tomasi c. France du 27 août 1992, a posé un principe nouveau: l'intégrité physique d'une personne privée de sa liberté doit bénéficier d'une garantie absolue, en raison de l'état d'infériorité dans lequel elle se trouve ${ }^{59}$, même si la Cour reconnaît par ailleurs que l'utilisation de la force peut être nécessaire pour assurer la sécurité en prison, pour maintenir l'ordre ou pour prévenir la commission d'infractions ${ }^{60}$. On constate aujourd'hui, tant en droit européen qu'en droit interne, des évolutions notables qui permettent l'encadrement de la violence en prison, ainsi que l'interdiction de certaines pratiques pouvant présenter des caractéristiques indignes.

Depuis cette nouvelle ligne jurisprudentielle, la Cour européenne des droits de l'homme a rendu un certain nombre de décisions qui visent toutes à limiter en détention l'usage de la force et à faire appliquer le principe de proportionnalité ${ }^{61}$. L'arrêt Alboréo c. France du 20 octobre 2011, qui a abouti à une condamnation de la France pour traitements inhumains et dégradants suite à l'usage excessif de la force contre un détenu lors de l'intervention d'agents d'une équipe régionale d'intervention et de sécurité (ERIS), constitue un bon exemple de ces nouvelles exigences ${ }^{62}$. Plus récemment, dans leur arrêt Karabet et autres c. Ukraine du 17 janvier 2013 relatif à la répression particulièrement violente d'un mouvement de protestation collective précédemment commise dans l'établissement, les juges européens ont rappelé que

[...] l'usage gratuit de la violence par les autorités visant à écraser un mouvement de protestation, à punir des prisonniers pour leur participation à un mouvement pacifique de grève de la faim et à écraser dans l'œuf toute velléité de plainte $[\ldots]$ constitue un traitement qui ne peut être qualifié que de torture ${ }^{63}$.
On retrouve une telle analyse dans l'arrêt Tali c. Estonie du 13 février 2014 ( $n^{\circ}$ 66393/10), qui rappelle avec fermeté que l'utilisation d'un spray au gaz poivre dans un espace confiné entraîne des effets particulièrement néfastes pour la santé, et ne constitue pas un moyen approprié pour immobiliser un détenu, à partir du moment où les gardiens pouvaient employer d'autres moyens moins dangereux. L'utilisation disproportionnée de la force est également au cœur du constat de violation de l'article 3 de la Convention de 1950 effectué par la Cour dans son arrêt Sapožkovs c. Lettonie du 11 février 2014, concernant un détenu violemment battu lors d'un transfert entre deux établissements ${ }^{64}$. Certains instruments autrefois utilisés traditionnellement en détention comme moyens de contrainte des corps ne sont désormais plus considérés comme admissibles: ainsi en est-il d'un lit de contention, dont l'usage doit être strictement justifié par les circonstances et ne peut en aucun cas constituer une punition ${ }^{65}$. Il est également inadmissible d'entraver une détenue pendant son séjour à la maternité (et plus particulièrement pendant la phase des contractions et juste après son accouchement) ${ }^{66}$, ou de nourrir de manière forcée un détenu sans nécessité médicale ${ }^{67}$. Ces arrêts se situent dans la même ligne que la loi pénitentiaire française, qui précise dans son article 44 que « [l]'administration pénitentiaire doit assurer à chaque personne détenue une protection effective de son intégrité physique en tous lieux collectifs et individuels».

\section{B. L'importance du principe de dignité en prison}

En dehors de l'usage de la force, la jurisprudence européenne a également cherché à rendre certaines pratiques

59. Cour EDH, 27 août 1992, Tomasi c. France, nº 12850/87. Le juge De Meyer, dans son opinion séparée, explicite même encore un peu plus la portée de ce nouveau principe: "À l'égard d'une personne privée de sa liberté, tout usage de la force physique qui n’est pas rendu strictement nécessaire par son propre comportement porte atteinte à la dignité humaine et doit, dès lors, être considéré comme une violation du droit garanti par l'article 3 de la Convention». Comme l'a précisé plus récemment l'arrêt Đurđević c. Croatie du 19 juillet 2011 (n 52442/o9), les États doivent prendre des mesures afin d' "offrir une protection effective, notamment aux enfants et autres personnes vulnérables, et [ces mesures doivent] comporter des actions raisonnables destinées à prévenir les mauvais traitements dont les autorités avaient ou auraient dû avoir connaissance» ( $\$ 102)$.

60. Cour EDH, 12 avril 2007, Ivan Vassilev c. Bulgarie, $\mathrm{n}^{\circ}$ 48130/99.

61. Par ailleurs, ces mêmes juges européens exigent depuis leur arrêt Assenov c. Bulgarie du 28 octobre 1998 (n² 24760/94) que les autorités étatiques diligentent une enquête effective, rapide, complète et indépendante sur les allégations de mauvais traitements, sous peine de se voir automatiquement condamnées pour violation de l'article 3 de la Convention EDH, que les faits aient été prouvés ou non. Pour des applications récentes, voir, inter alia, Cour EDH, 3 juin 2014, Yiğitdoğan c. Turquie et Habimi et autres c. Serbie, $\mathrm{n}^{\circ}$ 72174/10 et no 19072/o8.

62. Cour EDH, 20 octobre 2011, Alboréo $c$. France, $n^{\circ}$ 51019/o8. Voir l'arrêté du ministère de la Justice du 24 avril 2012 portant règlement d'emploi des équipes régionales d'intervention et de sécurité de l'administration pénitentiaire, Bulletin officiel du ministère de la Justice, no $2012-04$, 30 avril 2012, JUSK1240026A.

63. Cour EDH, 17 janvier 2013, Karabet et autres c. Ukraine, $\mathrm{n}^{\circ} 38906 / 07$ et 52025/07, $\$ 332$. Dans cette affaire, les détenus concernés ont été sauvagement battus par des agents masqués et par des gardiens de prison (à tel point que certains d'entre eux se sont évanouis), étroitement menottés et contraints de se déshabiller et de se tenir dans des postures humiliantes; privés d'accès à l'eau et à la nourriture et exposés à de basses températures sans vêtements adéquats, ils ont également été transférés dans un fourgon surpeuplé et aucune assistance médicale effective ne leur a été prodiguée à un moment quelconque de l'opération. Voir aussi Cour EDH, 26 mai 2015, Songül İnce et autres c. Turquie, $\mathrm{n}^{\circ} 25595 / 08$ et $34252 / 10$.

64. Cour EDH, 11 février 2014, Sapožkovs c. Lettonie, $\mathrm{n}^{\circ}$ 8550/03. Voir également les arrêts Milić et Nikezićc. Monténégro du 28 avril 2015 ( $\mathrm{n}^{\circ} 54999 / 10$ et 10609/11), concernant des violences infligées par des gardiens lors de la fouille d'une cellule.

65. Ces techniques ne peuvent que servir à éviter des automutilations, ou protéger des codétenus ou assurer la sécurité de l'établissement. Voir, inter alia, Cour EDH, 16 décembre 2014, Dimcho Dimov c. Bulgarie, nº 57123/o8: la Cour condamne l'État pour avoir menotté à son lit (par les poignets et les chevilles) pendant neuf jours consécutifs (pendant lesquels il ne fut détaché que trois fois par jour pour aller aux toilettes et prendre ses repas) un détenu souffrant de troubles comportementaux et qui avait fait part aux autorités pénitentiaires de sa volonté de s'automutiler.

66. Cour EDH, 14 mars 2016, Korneykova et Korneykov c. Ukraine, $\mathrm{n}^{\circ}$ 5666o/12.

67. Malgré son refus conscient, la nourriture avait été introduite de force dans la bouche du requérant, menotté, au moyen d'un écarteur buccal et d'un tube en caoutchouc spécial inséré dans l'œsophage, ce qui constitue un acte de torture pour les juges strasbourgeois. Voir Cour EDH, 5 avril 2005, Nevmerjitsky c. Ukraine, n 54825/00: F. Sudre, G. Gonzalez, M. Levinet, H. Surrel, «Jurisprudence de la Cour européenne des droits de l'homme 2005", Revue du droit public, n 3, 2006, p. 795. Voir également Cour EDH (déc.), 7 mars 2013, Rappaz c. Suisse, nº 73175/10. 
pénitentiaires plus dignes et respectueuses du corps du détenu.

Ainsi en est-il notamment du complexe problème des fouilles qui, tout en constituant une technique indispensable au maintien de l'ordre et de la sécurité dans les établissements, peuvent dans certains cas se dérouler dans des conditions inacceptables et contraires aux exigences de l'article 3 de la Convention EDH. Cette question est ainsi au cœur de l'affaire Valašinas c. Lituanie du 24 juillet 2001 dans laquelle la Cour de Strasbourg juge qu'une fouille au corps et l'examen des parties génitales d'un détenu effectués en présence d'une femme sont constitutifs d'un traitement dégradant contraire à l'article 3. Menée dans de telles conditions, cette investigation a dû, en effet, laisser au requérant «des sentiments d'angoisse et d'infériorité, sources d'humiliation et de vexation ${ }^{68}$. Ne sont pas davantage compatibles avec les standards européens, des fouilles corporelles systématiques (dont une fouille «intégrale» hebdomadaire), imposées à un détenu (quel que soit son degré de dangerosité) pendant plus de six ans. Ne reposant sur aucune exigence de sécurité, ces mesures ont porté atteinte à la dignité humaine du requérant ${ }^{69}$. Ces arrêts ont été directement à l'origine de l'avancée législative portée par l'article 57 de la loi pénitentiaire française de 2009 qui prévoit désormais que la «nature» et la «fréquence» des fouilles doivent être «strictement adaptées [aux] nécessités [de sécurité des personnes et de maintien du bon ordre dans l'établissement] et à la personnalité des personnes détenues». Par ailleurs, « [l] es fouilles intégrales ne sont possibles que si les fouilles par palpation ou l'utilisation des moyens de détection électronique sont insuffisantes $[\ldots] »^{70}$.

Au-delà de la question des fouilles, la Cour européenne œuvre également pour un meilleur respect de l'intégrité corporelle de la personne en détention, comme le montre par exemple l'arrêt Yankov c. Bulgarie du 11 décembre 2003 $\left(\mathrm{n}^{\circ} 39084 / 97\right)$, dans lequel elle retient une violation de l'article 3 en raison du rasage du crâne du requérant suite à son placement en isolement cellulaire, compte tenu de son âge ( 55 ans), et du fait que quelques jours plus tard il avait à comparaître en audience publique. La juridiction européenne a tenu en l'espèce à souligner qu'il s'agit de la modification forcée de l'apparence d'une personne dont celle-ci porte la marque, visible immédiatement par autrui et pendant un certain temps, qui constitue une pratique violant l'article 3 de la Convention EDH. Dans un arrêt Biržietis c. Lituanie rendu le 14 juin 2016 ( $n^{\circ}$ 49304/o9), elle a également jugé que l'interdiction absolue faite à un détenu de laisser pousser sa barbe (indépendamment de toute considération hygiénique ou autre) pendant qu'il purgeait une peine privative de liberté constitue une ingérence disproportionnée dans le respect du droit à la vie privée de l'intéressé, ne pouvant se justifier ni par des objectifs de défense de l'ordre et de la prévention de la criminalité en prison, ni par un quelconque besoin social impérieux. C'est même le refus de l' «éclat des supplices» évoqué par Foucault ${ }^{71}$ qui est désormais pris en compte par la Cour qui a eu l'occasion de préciser très récemment que la pratique consistant à mettre les personnes en détention provisoire dans des cages de métal au cours des audiences pendant leur procès constitue un traitement dégradant injustifiable, contraire à la dignité humaine ${ }^{72}$, qui viole donc l'article 3 de la Convention EDH.

La volonté contemporaine de ne plus cantonner la privation de liberté carcérale à un outil d'affliction et de souffrance et d'en faire également un outil permettant « de préparer l'insertion ou la réinsertion de la personne détenue afin de lui permettre de mener une vie responsable et de prévenir la commission de nouvelles infractions ${ }^{73}$ a totalement transformé le rapport au corps de celui qui est incarcéré. Certes, les conditions indignes de détention dans de nombreux établissements pénitentiaires ${ }^{74}$, la surpopulation carcérale, la violence interne à ces institutions rendent ces objectifs en partie virtuels ou inappliqués. Mais il est incontestable que l'émergence du principe de dignité, l'abandon de la

68. Cour EDH, 24 juillet 2001, Valašinas c. Lituanie, $\mathrm{n}^{\circ}$ 44558/98, \$ 117. Voir M. Herzog-Evans, «Tendances actuelles du droit des fouilles corporelles», Gazette du Palais, n 57, 26 février 2002, p. 3.

69. Cour EDH, 12 mars 2003, Öcalan c. Turquie, n 46221/99, \$ 232. Pour d'autres applications voir, inter alia, Cour EDH, 12 juin 2007, Frérot c. France, $\mathrm{n}^{\circ} 70204 / 01 ; 28$ octobre 2014, Ślusarczyk c. Pologne, $\mathrm{n}^{\circ}$ 23463/04; 15 septembre 2015, Milka c. Pologne, $\mathrm{n}^{\circ}$ 14322/12.

70. Le Conseil d'État a rappelé que si «les nécessités de l'ordre public et les contraintes du service public pénitentiaire peuvent légitimer l'application aux détenus d'un régime de fouilles corporelles intégrales, [...] l'exigence de proportionnalité des modalités selon lesquelles ces fouilles sont organisées implique qu'elles soient strictement adaptées [...] à la personnalité des personnes détenues qu'elles concernent» (CE, ord., 6 juin 2013, Section française de l'observatoire international des prisons, $\mathrm{n}^{\circ} 368816$ et juge des référés, 6 juin 2013, M. E., n 368875 ). Toutefois, l'article 111 de la loi n 2016-731 du 3 juin 2016 renforçant la lutte contre le crime organisé, le terrorisme et leur financement, et améliorant l'efficacité et les garanties de la procédure pénale a récemment ajouté que «[l]orsqu'il existe des raisons sérieuses de soupçonner l'introduction au sein de l'établissement pénitentiaire d'objets ou de substances interdits ou constituant une menace pour la sécurité des personnes ou des biens, le chef d'établissement peut également ordonner des fouilles dans des lieux et pour une période de temps déterminés, indépendamment de la personnalité des personnes détenues. Ces fouilles doivent être strictement nécessaires et proportionnées. Elles sont spécialement motivées et font l'objet d'un rapport circonstancié transmis au procureur de la République territorialement compétent et à la direction de l'administration pénitentiaire».

71. M. Foucault, Surveiller et punir..., p. 36.

72. Cour EDH, 17 juillet 2014, Svinarenko et Slyadnev c. Russie, nº 32541/o8.

73. Art. $1^{\text {er }}$ de la loi pénitentiaire française de 2009.

74. Attestées par la très volumineuse jurisprudence de la Cour européenne des droits de l'homme en la matière. Voir la fiche thématique de la Cour "Conditions de détention et traitement des détenus", avril 2017, en ligne: http://www.echr.coe.int/Documents/FS_Detention_conditions_FRA.pdf. Voir également les différents rapports du Comité européen pour la prévention de la torture et des peines ou traitements inhumains ou dégradants (CPT; http://www.cpt.coe.int/fr). Au plan interne, voir les rapports annuels du Contrôleur général des lieux de privation de liberté (http://www.cglpl.fr). 
conception du détenu-machine, ou encore la prise en compte du détenu-patient sont autant de marqueurs de cette évolution. Il n'en reste pas moins que le corps des personnes incarcérées reste encore aujourd'hui marqué par des particularités inhérentes à la détention. L'utilisation du corps comme moyen de lutte ou de revendication en est la preuve la plus éclatante. Qu'il s'agisse des grèves de la faim $^{75}$, des automutilations ${ }^{76}$, ou même des autolyses ${ }^{77}$, la prison revêt toujours les caractéristiques d'une institution pratiquant l' "investissement disciplinaire des corps » (pour citer une dernière fois Michel Foucault ${ }^{78}$...) auquel ces corps protestataires et révoltés font écho.

75. À l'époque contemporaine, suite à la décision des autorités britanniques de supprimer le statut spécial accordé aux détenus politiques irlandais à partir de 1976, dix détenus sont morts en prison, parmi lesquels Bobby Sands en 1981. En Allemagne, par l'utilisation des grèves de la faim, les membres de la Rote Armee Fraktion (RAF) ont réclamé à partir de 1972 une amélioration de leurs conditions de détention et notamment la fin des techniques de privation sensorielle. En France, le mouvement de grève de la faim le plus vaste remonte à la période 1957-1960, lors de l'emprisonnement des Nord-Africains pendant les événements d'Algérie (le point culminant étant atteint en 1961, avec une grève de la faim massive de mille trois cents détenus conduite par Ahmed Ben Bella, pour demander l'application du régime «A»). Quelques années plus tard, en 1971, quatre-vingt-dix détenus d'extrême gauche décident de faire une grève de la faim illimitée pour obtenir un régime spécial de détenu politique. Plus près de nous, les prisons turques ont connu dans les années 2000 des mouvements massifs de grèves de la faim pour protester contre la mise en place des prisons de type « $\mathrm{F} »$, limitant l'espace alloué aux détenus.

76. Pour Fabrice Fernandez, «L'individu au corps automutilé en prison semble se réapproprier (à l'instar de bon nombre de détenus) cette mémoire collective élaborée autour du sentiment d'oppression et de domination, qui dépasse le seul cadre carcéral mais dont il reste un élément emblématique» (F. Fernandez, «Du "corps otage" au "corps mémoire": les actes d'automutilation en prison chez les usagers de drogues et leurs mises en récits", Face à face, n 5, 2003, en ligne: https://faceaface.revues.org/421).

77. Selon une étude récente de l'Institut national des études démographiques (INED), dans les prisons françaises, près d'un décès sur deux est un suicide (INED, «Le suicide en prison», en ligne: https:/www.ined.fr/fr/tout-savoir-population/memos-demo/focus/suicide-en-prison).

78. M. Foucault, Surveiller et punir..., postface. L'auteur conclut son ouvrage par ces mots: «Dans cette humanité centrale et centralisée, effet et instrument de relations de pouvoir complexes, corps et forces assujettis par des dispositifs d'incarcération" multiples, objets pour des discours qui sont eux-mêmes des éléments de cette stratégie, il faut entendre le grondement de la bataille» (p. 315). 